\section{Heart beats fat}

\section{By Lev Osherovich, Senior Writer}

Researchers from The University of Texas Southwestern Medical Center have discovered that knocking down microRNA-208a in the heart leads to systemic benefits in obesity and metabolic disease, in addition to the already known effects on preventing cardiac hypertrophy and fibrosis. ${ }^{1}$ The findings could expand the potential indications for miRagen Therapeutics Inc.'s lead candidate, antimiR-208, which is partnered with Servier and is in preclinical development for cardiac hypertrophy.

Team leader Eric Olson, chairman of molecular biology at UT Southwestern and a cofounder and chief scientific advisor to miRagen, said his group initially set out to study miR-208a's function in the heart but ended up uncovering a mechanism by which the heart regulates systemic metabolism.

"We didn't go into this anticipating that the heart regulated metabolism-it wasn't what we were looking for," said Olson.

Now, miRagen thinks targeting cardiac miR-208a could help treat metabolic disease in patients with heart disease and mitigate cardiovascular risk in patients with metabolic disease. The company is expanding its preclinical testing of antimiR-208 into mouse obesity models.

\section{Heart miR miR}

Olson's team previously found that miR-208a was expressed in the heart, in which it negatively regulates the expression of a range of genes. ${ }^{2}$ As a follow-up to that work, the team used antimiR-208, a locked nucleic acid (LNA) oligomer, to study the effect of knocking down miR-208a.

Mice receiving a subcutaneous injection of antimiR-208 were leaner than animals given injection of an untargeted control antimiR. The slimming effect of miR-208a knockdown was even more pronounced when the mice were fed a high-fat diet. In overfed animals, antimiR-208 decreased weight gain, white adipose tissue hypertrophy and insulin resistance compared with a control antimiR.

Olson's team suspected that the effects of knocking down miR-208a stemmed from a key miR-208a-regulated gene called MED13 (mediator complex subunit 13). Previously, the team had found that miR-208a knockdown led to increased levels of Med13 compared with normal miR-208a expression.

"Because MED13 was one of the strongest targets of miR-208a, we wanted to determine the effect of loss- and gain-of-function of MED13," said Olson.
Indeed, overfed mice engineered to overexpress Med13 resembled overfed mice treated with antimiR-208, suggesting the effects of knocking down miR-208a were likely due to increased levels of Med13.

Olson's team also found that Med 13 knockout specifically in the heart led to greater weight gain, fat deposits and insulin insensitivity than normal Med13 expression. Altogether, the findings implicate MED13 as a heart-specific regulator of systemic metabolism and point to miR-208a as a key controller of MED13 expression.

Results were published in Cell. The findings are patented and licensed to miRagen.

\section{Obesity opportunity}

The most pressing question raised by Olson's work is how MED13's activity within the heart can strongly influence systemic metabolism. Olson thinks cardiac tissue likely sends an endocrine signal that affects energy utilization by other tissues, but the identity of such a signaling molecule remains unknown.

Although the mechanism remains murky, "perhaps it's not so surprising that the heart is communicating with the energy stores of the body," said Olson. "The heart has a tremendous appetite for energy but doesn't store energy on its own. It makes sense that there would be a system for signaling between the heart and fat deposits."

Olson now is trying to identify the signaling molecules that bridge the heart and metabolic system and hopes to uncover whether MED13 regulates metabolism in other tissues besides the heart. He noted that although miR-208a is found exclusively in the heart, a very similar miRNA called miR-208b is expressed in other tissues, along with MED13. Because miR-208a and miR-208b are so similar, it is possible that knocking down miR-208a could have effects on the periphery as well as the heart.

"We don't know what the signal is from the heart to the other tissues," said Olson. "We know that the effects of MED13 emanate from the heart, but this doesn't rule out that MED13 doesn't have roles in other tissues."

Olson noted that the effects of miR-208a on cardiac hypertrophy and fibrosis are likely distinct from its effects on metabolic disease, as MED13 does not appear to be necessary for antimiR-208's effect on cardiac hypertrophy.

This observation, said Olson, indicates there are likely additional miR-208a target genes involved in pathological cardiac remodeling, but the identity of those genes is not yet known.

miRagen CEO, president and cofounder William Marshall said the company had already been looking at antimiR-208 to treat cardiac hypertrophy with preserved ejection volume, a form of heart disease that is commonly caused by metabolic syndrome and obesity.

The new findings open the possibility of pursuing a metabolic disease label.

Marshall said that antimiR-208 has a favorable preclinical safety profile in two animal models of cardiac hypertrophy and that the compound's effect on heart strength should, if anything, reduce 


\section{ANALYSIS}

cardiovascular event risk in obese or diabetic patients.

"Our data show a direct benefit to heart health with this compound, suggesting that liability to cardiovascular problems is not going to be an issue," said Marshall.

He added that Olson's findings "imply that antimiR-208 could be used for patients with obesity and metabolic syndrome but who don't necessarily yet have cardiac hypertrophy." This hypothesis could be tested by stratifying patients based on both their weight and the state of their hearts.

Last year, miRagen partnered with Servier to co-develop antimiR-208 and two other therapeutic candidates for cardiovascular indications. miRagen's LNA antisense technology is licensed from Santaris Pharma A/S.

In April, miRagen closed a $\$ 20$ million series B round.
Osherovich, L. SciBX 5(18); doi:10.1038/scibx.2012.455

Published online May 3, 2012

\section{REFERENCES}

1. Grueter, C.E. et al. Cell; published online April 27, 2012; doi:10.1016/j.cell.2012.03.029

Contact: Eric N. Olson, The University of Texas Southwestern Medical Center, Dallas, Texas e-mail: eric.olson@utsouthwestern.edu

2. van Rooij, E. et al. Science 316, 575-579 (2007)

COMPANIES AND INSTITUTIONS MENTIONED miRagen Therapeutics Inc., Boulder, Colo. Santaris Pharma A/S, Horsholm, Denmark Servier, Neuilly-sur-Seine, France The University of Texas Southwestern Medical Center, Dallas, Texas 